\title{
Recording and self-enhancement of dynamic polarization gratings in degenerate four-wave-mixing geometry
}

\author{
A.Ozols*, A.Belyaev, V.Kokars, P.Augustovs, K.Traskovskis, D.Saharov \\ Faculty of Material Science and Applied Chemistry, Riga Technical University, 7/3 Paula Valdena \\ iela, Riga, Latvia, LV-1007
}

\begin{abstract}
Scalar and vectorial degenerate four-wave-mixing (DFWM) in azobenzene molecular K-RJ-4-3 [N,N-bis(5,5,5tryphenylpentyl)-4-((4-tritylphenyl)diazenyl)aniline] and in chalcogenide a- $\mathrm{As}_{2} \mathrm{~S}_{3}$ glassy films is experimentally investigated. A coherent self-enhancement (CSE) of holographic gratings (HG) in this geometry was experimentally confirmed, for the first time to our knowledge, thus establishing a new method of HG recording, the DFWM CSE recording. Scalar linear $s-s, p-p$ and circular R-R(right) polarizations and orthogonal linear $s-p$ and circular L(left)-R polarizations were used for $\mathrm{HG}$ recording with $2 \mu \mathrm{m}$ period at $532 \mathrm{~nm}$. In the case of K-RJ-4-3 film L-R polarizations were the most efficient enabling the maximum DFWM efficiency $\rho_{\max }=14.5 \%$ whereas in the case of a- $\mathrm{As}_{2} \mathrm{~S}_{3}$ film $s-s$ polarizations were the best with $\rho_{\max }=6.0 \%$. DFWM CSE recording has exhibited a different polarization dependence compared to normal DWFM recording. CSE factor $\rho / \rho_{0}\left(\rho_{0}\right.$ being the initial $\rho$ ) was the highest for K-RJ-4-3 film (6.8) with $s-s$ polarizations compared to 3 for a- $\mathrm{As}_{2} \mathrm{~S}_{3}$ film with $p$ - $p$ polarizations. Two-wave HG recording was also studied for comparison.
\end{abstract}

Keywords: dynamic holography, polarization gratings, degenerate four-wave mixing, self-enhancement of gratings, two-wave mixing

\section{INTRODUCTION}

Degenerate four-wave- mixing (DFWM) is a nonlinear optical process which involves the interaction of two pump waves in the medium with a probe wave to generate the fourth phase-conjugated wave-the signal wave ${ }^{1}$. From the point of view of holography, one can say that two pump waves record a dynamic holographic grating (HG) in a photosensitive material, and the probe wave diffraction from this HG creates the signal wave ${ }^{2}$. In fact, the DFWM process is more complicated because reflection HG are also recorded by the interference of the probe wave with both pump waves. Thus DFWM can also be defined as an unusual method of HG recording which enables real-time phase conjugation of light beams. Holographic description of DFWM is often more convenient and it will be used also in our paper.

DFWM has found a variety of applications in different fields of science and technology. One can mention nonlinear spectroscopy ${ }^{1,3,4}$, optical information processing (e.g., linear operations of image convolution and correlation, nonlinear operation of image edge detection) ${ }^{4,5}$, distortion correction in adaptive optics ${ }^{5}$ (e.g., laser beam aiming in nonhomogenous media ${ }^{4}$, image distortion correction in telescopes ${ }^{6}$, direct image transmission through optical fibres ${ }^{7,8}$ ) ,focusing beyond the diffraction limit ${ }^{9}$, optimization of volume holographic memory ${ }^{10}$.

Usually scalar DFWM is studied when the polarizations of all interacting waves are parallel, and scalar dynamic gratings are recorded in the photosensitive material ${ }^{1,2,4,5,6}$ if we use the holographic description of DFWM. From the point of view of fundamental science as well of applications the vectorial DFWM is of large interest when the pump and probes waves have different polarizations. Then polarization dynamic gratings are recorded. As far as we know, vectorial DFWM has been studied mainly theoretically ${ }^{11,12}$. Experimental papers are rather rare ${ }^{13}$. This was the first part of the motivation behind this paper devoted to experimental studies of scalar and vectorial DFWM in $\mathrm{a}-\mathrm{As}_{2} \mathrm{~S}_{3}$ chalcogenide and in azobenzene molecular K-RJ-4-3 [N,N-bis(5,5,5-tryphenylpentyl)-4-((4-tritylphenyl)diazenyl)aniline] glassy films . Previously we have experimentally studied DFWM in amorphous chalcogenide $\mathrm{As}_{40} \mathrm{~S}_{15} \mathrm{Se}_{45}$ and $\mathrm{As}_{2} \mathrm{~S}_{3}$ films ${ }^{14,15}$ and in azobenzene oligomer 4-((4-nitrophenyl)diazenyl)-N,N-bis(2-(tetrahydro-2H-pyran-2-yloxy)ethyl)benzenamine films ${ }^{15}$.

*aozols@latnet.lv; phone +371-29449921; fax +371-67089074

Eighth International Conference on Advanced Optical Materials and Devices (AOMD-8), edited by Janis Spigulis, Proc. of SPIE Vol. 9421, 942108 · ( 2014 SPIE · CCC code: 0277-786X/14/\$18 · doi: 10.1117/12.2084046 
The second part of the motivation behind this paper was to study the coherent self-enhancement (CSE) of dynamic HG in scalar and vectorial DFWM geometry. Self-enhancement ( SE) of a nonstationary hologram is the increase in its diffraction efficiency (DE) over time under the stimulus of a single beam light irradiation or simply in the dark ${ }^{16}$. Three types of SE can be distinguished: i) CSE due to the holographic recording by diffracted waves; ii) incoherent SE due to the contrast and/or transmission increase of a hologram by incoherent light; iii) relaxational (or dark) SE due to the contrast and/or transmission increase of a hologram by thermostimulated relaxation processes. All three SE types can take place simultaneously. SE can also be regarded as a two-stage hologram recording method, which is profitable when the recording energy or exposure time is limited at the first stage. Recently we have experimentally investigated SE of scalar and vector HG in azobenzene molecular glassy films ${ }^{16}$. However, to our best knowledge, nobody has explored SE in DFWM geometry. It was not even clear whether it was possible at all.

In this paper, scalar and vectorial DFWM in K-RJ-4-3 azobenzene molecular and in a- $\mathrm{As}_{2} \mathrm{~S}_{3}$ chalcogenide glassy films are experimentally investigated. Thus dynamic polarization transmission and reflection HG were recorded in general case by spatially periodic light intensity and light polarization distribution. CSE of HG in this geometry was studied as well. Exposure time dependences were measured. Strong but different DFWM polarization dependences in both films were found. It was also shown that CSE of HG in DFWM geometry is also possible but not in all cases. DFWM CSE polarization dependences almost completely coincided with those of DFWM. For comparison, self-diffraction (SDE) exposure time dependences for usual two-wave HG recording were measured before the DFWM experiments. SDE and DFWM efficiency polarization dependences were rather different.

\section{SAMPLES AND EXPERIMENTS}

Azobenzene molecular K-RJ-4-3 [N,N-bis(5,5,5-tryphenylpentyl)-4-((4-tritylphenyl)diazenyl)aniline](Fig.1) and a$\mathrm{As}_{2} \mathrm{~S}_{3}$ glassy films were used for experiments. Their thickness was $2 \mu \mathrm{m}$ and $12 \mu \mathrm{m}$, respectively. K-RJ-4-3 film was synthesized and spin-coated onto the glass substrate in our Faculty whereas a- $\mathrm{As}_{2} \mathrm{~S}_{3}$ (shortly As-S) film (thermally evaporated onto the glass substrate with the band-gap of $2.38 \mathrm{eV}$ corresponding to $521 \mathrm{~nm}$ ) was provided by Dr.J. Teteris from the Institute of Solid State Physics, University of Latvia. The size of samples was $7 \times 7 \mathrm{~cm}^{2}$.

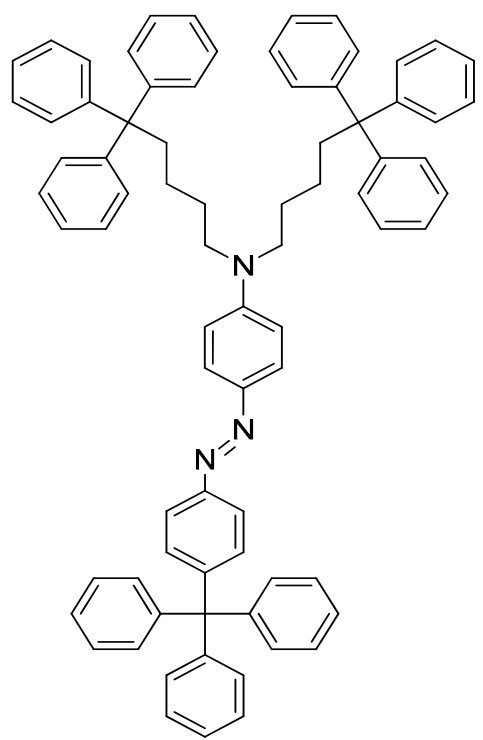

Fig.1.Chemical structure of K-RJ-4-3 molecule. This is a push-pull molecule of donor-acceptor type enabling intramolecular charge transfer and trans-cis photoisomerization. Such molecules in the film are bound together by van der Waals forces. The long wavelength (trans) absorption maximum is located at 457 $\mathrm{nm}$ in $\mathrm{CH}_{2} \mathrm{Cl}_{2}$ solution.

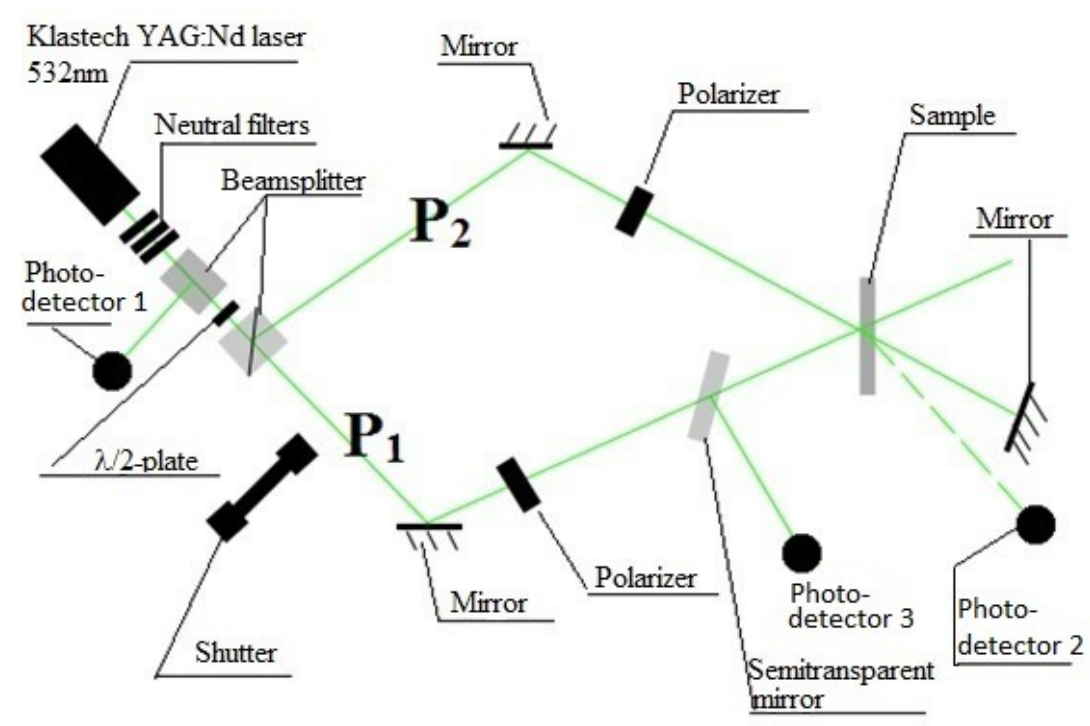

Fig.2. Experimental setup for $\mathrm{HG}$ recording in DFWM geometry and for CSE measurements. $P_{1}$ and $P_{2}$ are the incident pump waves. The probe wave arises by the reflection of transmitted $\mathrm{P}_{2}$ beam from the mirror behind the sample. Photodetector 1 was monitoring the laser power, photodetector 2 was measuring SDE, photodetector 3 was measuring the inverted (signal) wave power. DFWM measurement with orthogonal $s-p$ recording polarizations in $\mathrm{P}_{1}$ and $\mathrm{P}_{2}$ beams, respectively, is shown. In CSE experiments the shutter blocked the $\mathrm{P}_{1}$ beam. In the case of SDE measurements the mirror behind the sample was removed and photodetector 2 measured the diffracted wave power. To change the polarizations of pump waves additional $\lambda / 2$ and $\lambda / 4$ plates were inserted in $P_{1}$ and $P_{2}$ beams. 
The layout of the experimental setup is shown in Fig.2. KLASTECH DENICAFC 532-300 CW diode pumped solid state laser irradiating vertically polarized (s-polarization) $532 \mathrm{~nm}$ green light was used as a highly coherent (coherence length about $100 \mathrm{~m}$ ) light source. Transmission $\mathrm{HG}$ period recorded by $\mathrm{P}_{1}$ and $\mathrm{P}_{2}$ beams was $2.0 \mu \mathrm{m}$. Recording light intensity was $I=0.90 \mathrm{~W} / \mathrm{cm}^{2}$ (with respect to $1 / \mathrm{e}^{2}$ beam diameter of $1.47 \mathrm{~mm}$ ). The recording beam ratio (i.e., the ratio $P_{2} / P_{1}$ just before the sample) was 3.0 corresponding to the interference fringe visibility of 0.866 . This ratio is not optimal for normal HG recording (and SDE) but it is optimal to maximize the inverted beam (signal beam) power $P_{i n v}$ as shown by our calculations which will be published elsewhere. Signals from photodetectors were continuously measured as the functions of exposure time $(t)$ by OPHIR Nova II power meter and stored in the PC memory. All the experiments were carried out at room temperature.

The experimental procedure consisted of three parts. First, to evaluate the recording efficiency at each polarization pair SDE time dependence was measured in normal holographic recording (two-wave-mixing) geometry. SDE is a ratio of the first-order diffracted beam power to the power of the more distant recording beam when both recording beams $\left(\mathrm{P}_{1}\right.$ and $\mathrm{P}_{2}$ as in Fig.2) are present ${ }^{16}$. In our experiments, SDE was the ratio of the diffracted power measured by the photodetector 2 and the pump beam power $P_{2}$. From these measurements three quantities were found characterizing the recording efficiency-SDE $\max$ [ measured in\%], specific recording energy $W_{\max }=(I t)_{\max } / \mathrm{SDE}_{\max }\left[\operatorname{measured~in~} \mathrm{J} /\left(\mathrm{cm}^{2} \%\right)\right]$ and recording efficiency factor $\mathrm{REF}_{\mathrm{SDE}}=\mathrm{SDE}_{\max } / W_{\max }\left[(\% \mathrm{~cm})^{2} / \mathrm{J}\right]$. This last combined factor seems preferable compared to $W_{\max }$ because the role of $\mathrm{SDE}$ (and thus of signal-to-noise ratio) is accented.

Second, DFWM efficiency time dependences were measured for each pair of polarizations. DFWM efficiency was defined as $\rho=P_{\text {inv }} / P_{I}[\%]$. As in the SDE case, $\rho_{\max }[\%]$, specific recording energy $H_{\max }=(I t)_{\max } / \rho_{\max }\left[\mathrm{J} /\left(\mathrm{cm}^{2} \%\right)\right]$ and $\mathrm{REF}_{\mathrm{DFWM}}=\rho_{\max } / H_{\max }\left[(\% \mathrm{~cm})^{2} / \mathrm{J}\right]$ were found.

Third, CSE in DFWM geometry was studied. HG was recorded until small DFWM efficiency value about $0.1 \rho_{\max }$ as in the second part of experiments. After that, the beam $\mathrm{P}_{1}$ was blocked and the exposure proceeded only with one pump beam $\mathrm{P}_{2}$ (of course, the probe beam opposite to $\mathrm{P}_{2}$ was always present, except for SDE measurements) and DFWM efficiency was measured as in previous case. Again, $\rho_{\max }, H_{\max }$ and $\mathrm{REF}_{\mathrm{CSE}}$ have been determined. Besides, we have found also the CSE factor $\rho / \rho_{0}$ (where $\rho_{0}$ was the initial DFWM efficiency) as the function of exposure time. We estimate that SDE and $\rho$ were measured with $2.0 \%$ accuracy but the quantities $\rho / \rho_{0}, W_{\max }$ and $H_{\max }$, and REF were determined with $2.8 \%, 3.2 \%$ and $3.7 \%$ accuracy , respectively.

\section{RESULTS AND DISCUSSION}

Experimental results are presented in Figs.3-21 and in Tables 1-6. They consist of three parts corresponding to SDE, DFWM and DFWM CSE measurements. In Figs.3-8 SDE exposure time dependences for various recording beam polarization pairs are shown.

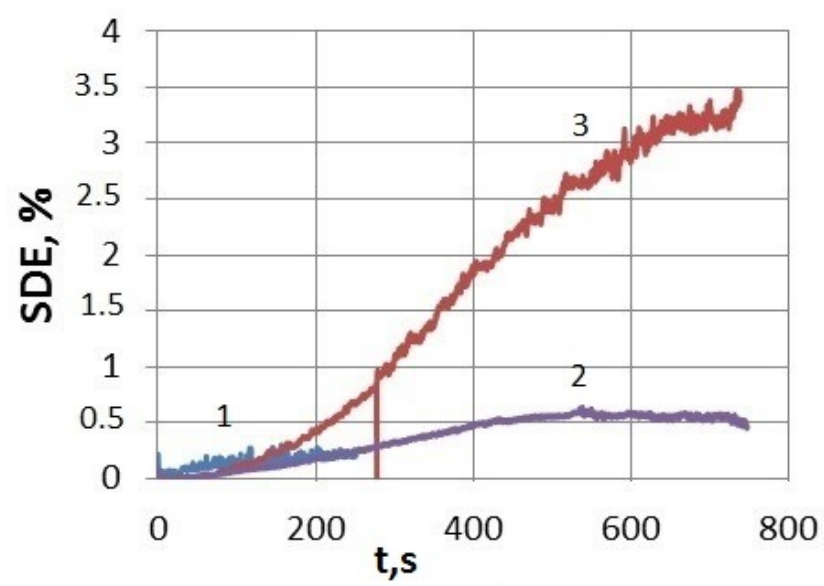

Fig.3. SDE exposure time dependences for sample K-RJ-4-3 in

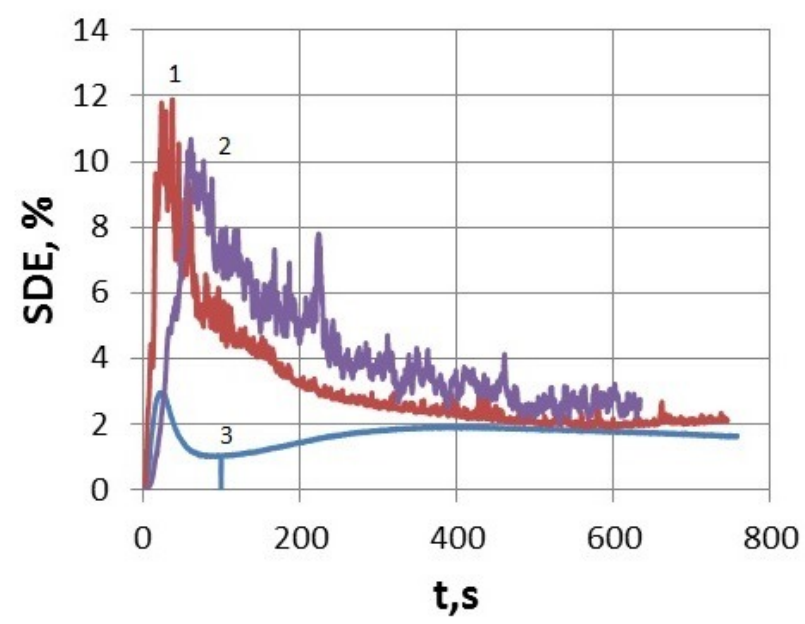

Fig.4. SDE exposure time dependences for sample As-S in the 
the case of scalar $s-s(1), \mathrm{R}-\mathrm{R}(2)$ and $p-p$ (3) recording beam polarizations.

\section{S-P K-RJ-4-3}

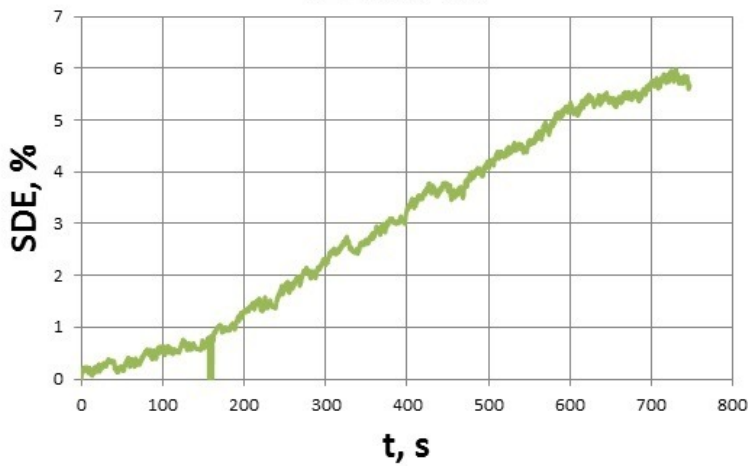

Fig.5. SDE exposure time dependences for sample K-RJ-4-3 in the case of orthogonal $s-p$ recording beam polarizations.

\section{L-R K-RJ-4-3}

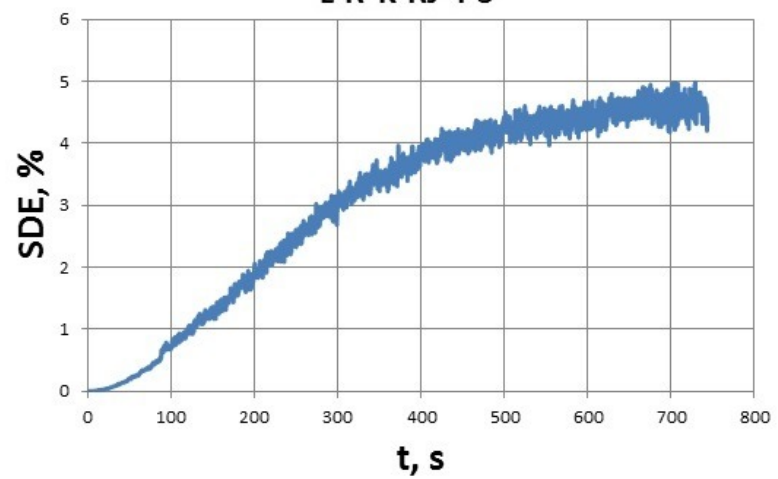

Fig.7. SDE exposure time dependences for sample K-RJ-4-3 in the case of orthogonal L-R recording beam polarizations. case of scalar $s-s(1), \mathrm{R}-\mathrm{R}(2)$ and $p-p$ (3) recording beam polarizations.

\section{S-P As-S}

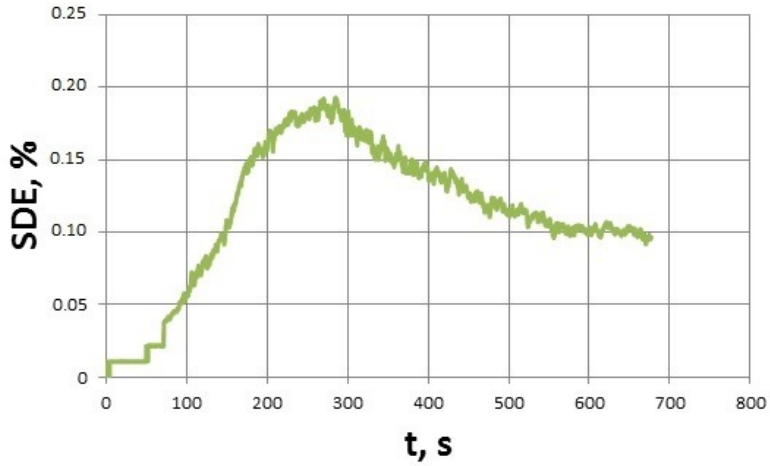

Fig.6. SDE exposure time dependences for sample As-S in the case of orthogonal $s-p$ recording beam polarizations.

\section{L-R As-S}

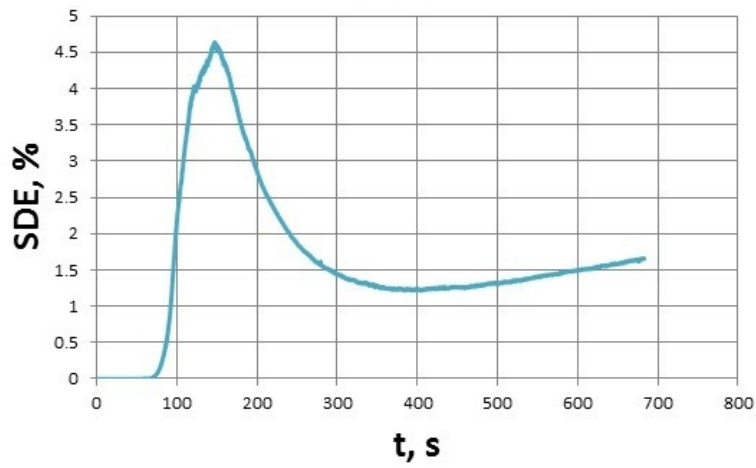

Fig.8. SDE exposure time dependences for sample As-S in the case of orthogonal L-R recording beam polarizations.

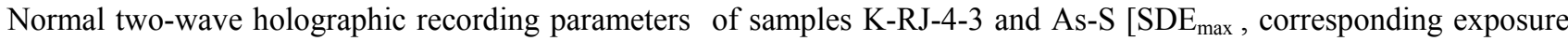
time $t_{\max }, W_{\max }$, and $\mathrm{REF}_{\mathrm{SDE}}$, see Section 2] are summarized in Table 1 and Table 2, respectively.

Table 1. Normal two- wave holographic recording parameters of sample K-RJ-4-3 for different recording beam polarization pairs.

\begin{tabular}{|c|c|c|c|c|c|}
\hline & $s-s$ & $p-p$ & $\mathrm{R}-\mathrm{R}$ & $s-p$ & $\mathrm{~L}-\mathrm{R}$ \\
\hline $\mathrm{SDE}_{\max }, \%$ & 0.30 & 3.4 & 0.60 & 5.8 & 725 \\
\hline$t_{\max ,} \mathrm{s}$ & 160 & 750 & 550 & 113 & 700 \\
\hline$W_{\max }, \mathrm{J} /\left(\mathrm{cm}^{2} \%\right)$ & 480 & 199 & 825 & $5.1 * 10^{-2}$ & $3.4^{*} 10^{-2}$ \\
\hline $\mathrm{REF}_{\mathrm{SDE}},(\% \mathrm{~cm})^{2} / \mathrm{J}$ & $6.3 * 10^{-4}$ & $1.7 * 10^{-2}$ & $7.3 * 10^{-4}$ & 25 \\
\hline
\end{tabular}

Using $\mathrm{REF}_{\mathrm{SDE}}$ criterion one can see from Table 1 that the most efficient polarization pair is orthogonal $s-p$. Then come $\mathrm{L}-\mathrm{R}, p-p, \mathrm{R}-\mathrm{R}$ and $s-s$ polarizations. 
Table 2. Normal two-wave holographic recording parameters of sample As-S for different recording beam polarization pairs.

\begin{tabular}{|c|c|c|c|c|c|}
\hline & $s-s$ & $p-p$ & $\mathrm{R}-\mathrm{R}$ & $s-p$ & L-R \\
\hline $\mathrm{SDE}_{\max }, \%$ & 11 & 3.0 & 10.8 & 0.19 & 260 \\
\hline$t_{\max ,} \mathrm{s}$ & 25 & 15 & 70 & 1230 & 29 \\
\hline$W_{\max }, \mathrm{J} /\left(\mathrm{cm}^{2} \%\right)$ & 2.0 & 4.5 & 5.8 & $1.5^{*} 10^{-4}$ & 0.16 \\
\hline $\mathrm{REF}_{\mathrm{SDE}},(\% \mathrm{~cm})^{2} / \mathrm{J}$ & 5.5 & 0.67 & 1.9 & \\
\hline
\end{tabular}

As in previous case, using $\mathrm{REF}_{\mathrm{SDE}}$ criterion one can see from Table 2 that the most efficient polarization pair is scalar $s$ $s$. Then come R-R, $p-p$, L-R and $s-p$. SDE polarization dependence now is markedly different compared to K-RJ-4-3 case. Besides, As-S sample exhibits much more efficient holographic recording than K-RJ-4-3.

Next,in Figs.9-12 DFWM efficiency exposure time dependences for various recording beam polarization pairs are shown.

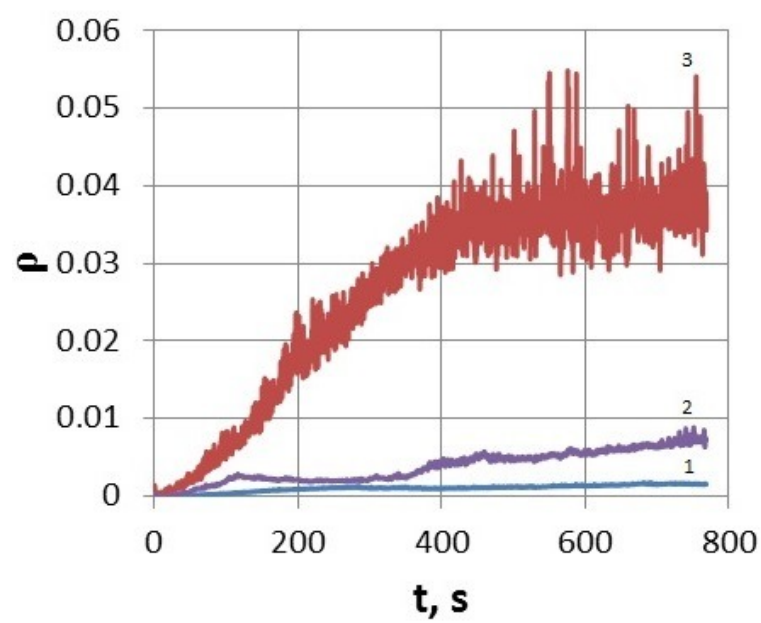

Fig.9. DFWM efficiency exposure time dependences for sample K-RJ-4-3 in the case of scalar $s-s(1), \mathrm{R}-\mathrm{R}(2)$ and $p-p$ (3) recording beam polarizations.

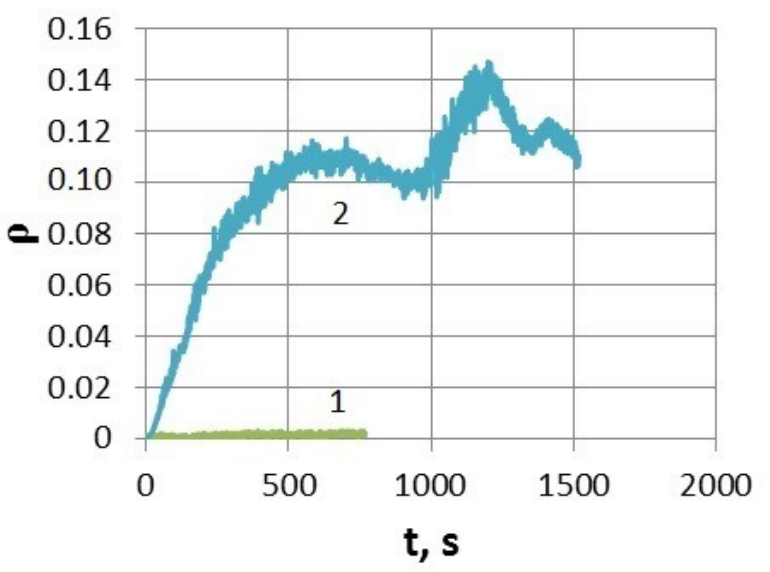

Fig.11. DFWM efficiency exposure time dependences for sample K-RJ-4-3 in the case of orthogonal $s-p$ (1) and L-R (2) recording beam polarizations.

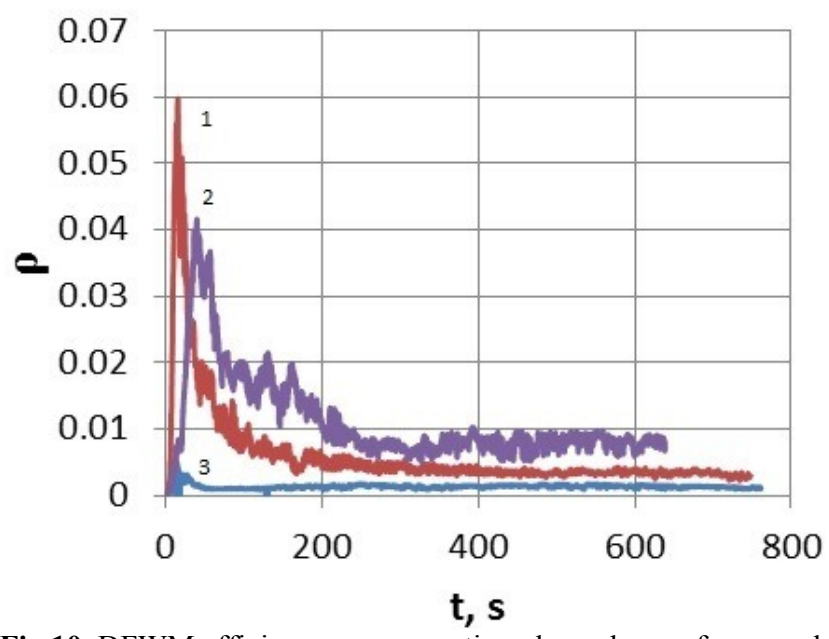

Fig.10. DFWM efficiency exposure time dependences for sample As-S in the case of scalar $s-s(1), \mathrm{R}-\mathrm{R}(2)$ and $p-p$ (3) recording beam polarizations.

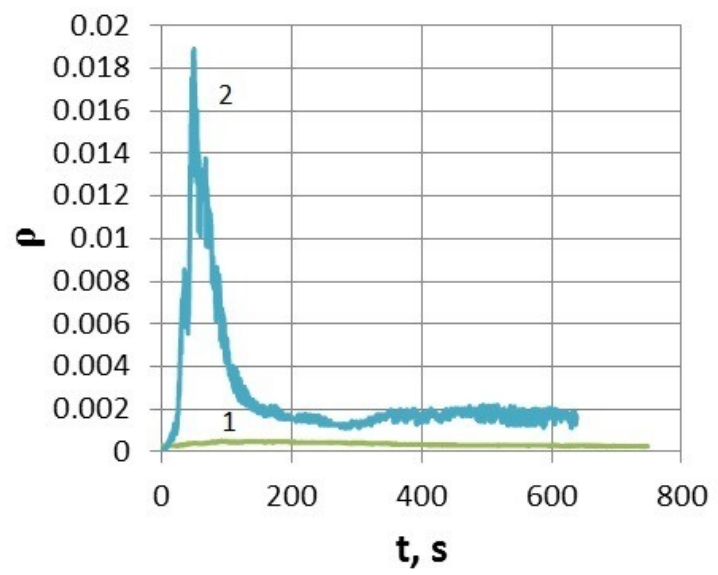

Fig.12. DFWM efficiency exposure time dependences for sample As-S in the case of orthogonal $s-p$ (1) and L-R (2) recording beam polarizations. 
The tendency can be seen when one compares Figs.3-8 and Figs.9-12 that DFWM efficiency time dependences have more pronounced maxima than those of SDE. Especially this tendency is pronounced in the case of As-S sample. Probably, this is the consequence of additional reflection HG recorded together with the main transmission HG by two pump waves $\left(\mathrm{P}_{1}, \mathrm{P}_{2}\right)$ and a probe wave (opposite to $\mathrm{P}_{2}$, Fig.2.), and with the stronger recording intensity dependence in the case of As-S sample ${ }^{15}$.

DFWM holographic recording parameters of samples K-RJ-4-3 and As-S [ $\rho_{\max }$, corresponding exposure time $t_{\max }, H_{\max }$ and $\mathrm{REF}_{\mathrm{DFWM}}$, see Section 2] are summarized in Table 3 and Table 4, respectively.

Table 3. DFWM holographic recording parameters of sample K-RJ-4-3 for different recording beam polarization pairs.

\begin{tabular}{|c|c|c|c|c|c|}
\hline & $s-s$ & $p-p$ & $\mathrm{R}-\mathrm{R}$ & $s-p$ & 0.23 \\
\hline$\rho_{\max }, \%$ & 0.17 & 3.8 & 0.75 & 700 & 14.5 \\
\hline$t_{\max ,} \mathrm{s}$ & 680 & 600 & 750 & 2740 & 75 \\
\hline$H_{\max }, \mathrm{J} /\left(\mathrm{cm}^{2} \%\right)$ & 3600 & 142 & 800 & $8.4^{*} 10^{-5}$ & 0.19 \\
\hline $\begin{array}{c}\mathrm{REF}_{\mathrm{DFWM}}, \\
(\% \mathrm{~cm})^{2} / \mathrm{J}\end{array}$ & $4.7^{*} 10^{-5}$ & $2.7 * 10^{-2}$ & $8.3^{-4} 10^{-4}$ & & \\
\hline
\end{tabular}

Using $\mathrm{REF}_{\mathrm{DFWM}}$ criterion one can see from Table 3 that the most efficient polarization pair is orthogonal L-R. Then come $p-p$, R-R, $s-p$ and $s-s$. Thus polarization dependence in DFWM case differs from SDE case (Table 1).

Table 4. DFWM holographic recording parameters of sample As-S for different recording beam polarization pairs.

\begin{tabular}{|c|c|c|c|c|c|}
\hline & $s-s$ & $p-p$ & R-R & $s-p$ & 0.05 \\
\hline$\rho_{\max }, \%$ & 6.0 & 0.50 & 4.2 & 100 & 48 \\
\hline$t_{\max ,,} \mathrm{s}$ & 20 & 10 & 40 & 1800 & 23 \\
\hline$H_{\max }, \mathrm{J} /\left(\mathrm{cm}^{2} \%\right)$ & 3.0 & 18 & 8.0 & $2.8^{*} 10^{-5}$ & $8.3^{*} 10^{-2}$ \\
\hline $\begin{array}{c}\mathrm{REF} \\
(\% \mathrm{~cm})^{2} / \mathrm{J}\end{array}$ & 2.0 & $2.7^{*} 10^{-2}$ & 0.53 & \\
\hline
\end{tabular}

According to $\mathrm{REF}_{\mathrm{DFWM}}$ criterion it follows from Table 4 that in the case of As-S sample the most efficient polarization pair is $s-s$. Then come R-R, L-R, $p-p$ and $s-p$. In contrast to K-RJ-4-3, this sequence exactly coincides with SDE polarization pair sequence for As-S.

Comparing Tables 3 and 4 one can see that again As-S sample is generally more efficient than K-RJ-4-3 sample .This is mainly due to the higher photosensitivity. However, the highest DFWM efficiency of $14.5 \%$ is achieved with K-RJ-4-3 sample and L-R polarizations. DFWM geometry allows to increase the recording efficiency in K-RJ-4-3 sample but not in As-S sample when compared with the SDE case.

How can one explain the different HG recording efficiencies of K-RJ-4-2 and As-S, the effects of recording light polarization and DFWM geometry? First of all, the recording mechanisms are different.

Previously ${ }^{16}$ we have come to the conclusion that HG recording in our organic molecular glasses at $532 \mathrm{~nm}$ is due to the azochromophore photoorientation in the process of trans-cis photoisomerization with a possible contribution of azochromophore photodegradation via polarization selective photoinduced N-N bond breaking. These processes are accompanied by mass transfer enabled by athermal fluidization. As the result, photoinduced changes in refractive index and absorption coefficient, as well as in surface relief are produced. The resulting HG is mainly the phase one. We believe that in our experiments only volume transmission HG mainly contribute since we worked in transmission mode.

HG recording in As-S in the case of band-gap or super-band-gap light is based on photoinduced structural canges (PSC). Various studies of different authors have shown that PSC in nonannealed As-S films are mainly due to the photoinduced breaking of ,wrong” As-As bonds followed by the phonon-assisted creation of As-S bonds ${ }^{17,18}$.PSC manifest themselves by changes of the absorption coefficient, refractive index, thickness, density, michrochardness and dissolution rate ${ }^{17}$.

From these mechanisms one can roughly expect that orthogonal polarizations are preferable in the case of K-RJ-4-3 because of more pronounced photoanisotropy whereas scalar (parallel) polarizations are preferable in the case of As-S. 
This conclusion is supported by the previously given data. Evidently, the difference in mechanisms and thicknesses (determining SDE and DFWM efficiency) is, most probably, responsible for different efficiency of both studied materials. As for recording efficiency dependence on recording polarizations, it is determined by material response.

Why the recording efficiency polarization dependence are different for normal (SDE) and DFWM recording in K-RJ-43 sample but the same in As-S sample? As mentioned above, this can be explained by a stronger effect of additional reflection HG in the case of K-RJ-4-3 sample. Generally, the main transmission HG and additional reflection HG are anisotropic polarization HG recorded by both periodic light intensity and light polarization distributions. The diffracted waves from all these dynamic HG interfere in a nonlinear medium. Further experimental and theoretical studies are needed to give definite answers to the above questions.

Holographic recording parameters, both in usual two-wave and DFWM geometry, obtained in our previous studies in aAs-S-Se ${ }^{14}, a_{-} \mathrm{As}_{2} \mathrm{~S}_{3}{ }^{15}$ and azobenzene oligomer ${ }^{15}$ films with red light $(632.8 \mathrm{~nm})$ were generally lower than those in this paper.

In the case of As-S and azobenzene oligomer films this was because of insufficient absorption.

Finally, let us consider our CSE results presented in Figs.13-21 and Tables 5,6. The vertical lines in Figs.13-21 denote the beginning of CSE process by one incident pump beam $\mathrm{P}_{2}$.

S-S K-RJ-4-3

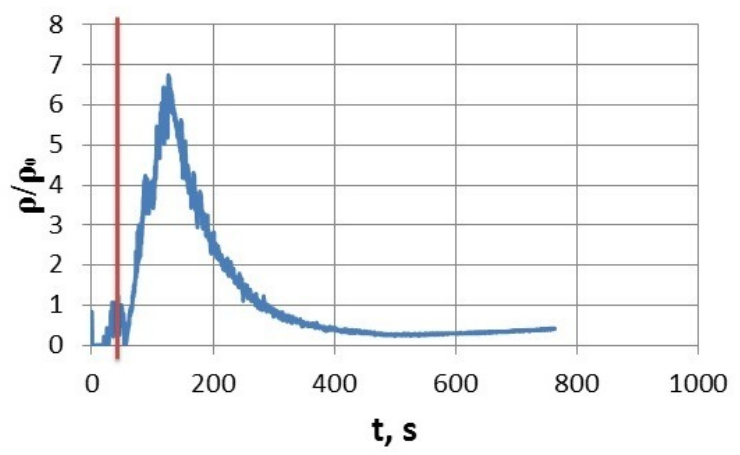

Fig.13. CSE factor exposure time dependence for K-RJ-4-3 sample in the case of $s-s$ recording polarizations.

\section{P-P K-RJ-4-3}

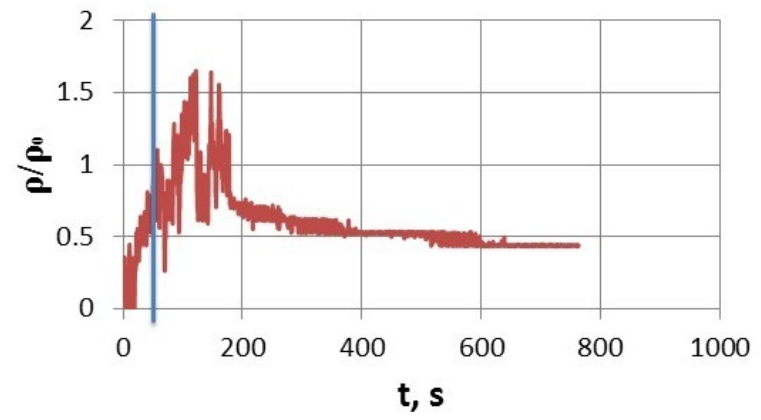

Fig.15. CSE factor exposure time dependence for K-RJ-43 sample in the case of $p$ - $p$ recording polarizations.

\section{S-S As-S}

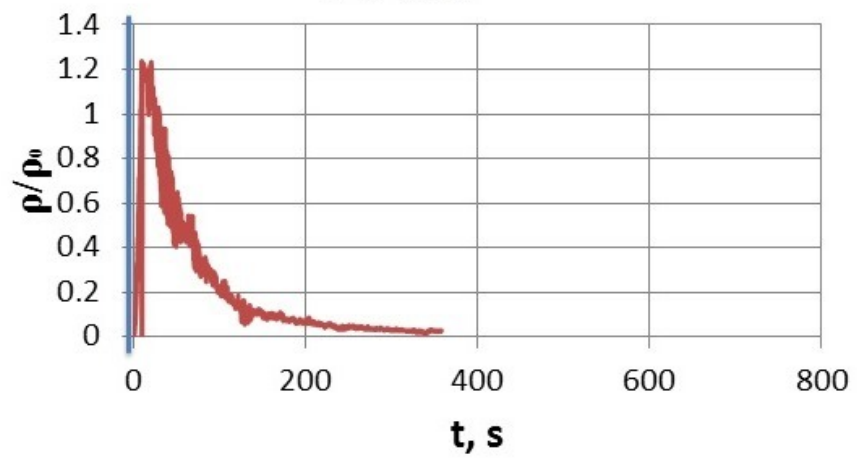

Fig.14. CSE factor exposure time dependence for As-S sample in the case of $s-s$ recording polarizations.

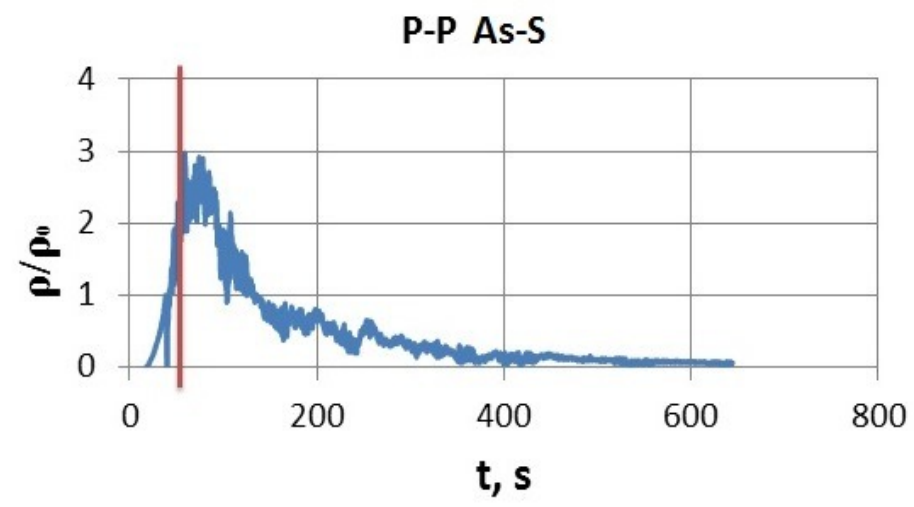

Fig.16. CSE factor exposure time dependence for As-S sample in the case of $p$ - $p$ recording polarizations.

One can see from CSE Figs.13-21 in comparison with DFWM Figs. 9-12 that CSE exposure time dependences generally have sharper maxima. CSE phenomenon can be explained by the model of complementary HG recorded by pump waves and diffracted waves ${ }^{16}$. The visibility of the main transmission HG in CSE process is much lower than in normal recording and DFWM cases because the diffracted $\mathrm{P}_{2}$ wave (Fig.2) substitutes the $\mathrm{P}_{1}$ wave, and the former is much 
stronger than the latter. However, no such sharpening effect is observed when CSE of normal HG takes place ${ }^{16}$. Evidently, again this is the effect of additional reflection HG recorded in DFWM process at the first stage, and complementary reflection $\mathrm{HG}$ recorded at the second stage of CSE.

\section{R-R K-RJ-4-3}

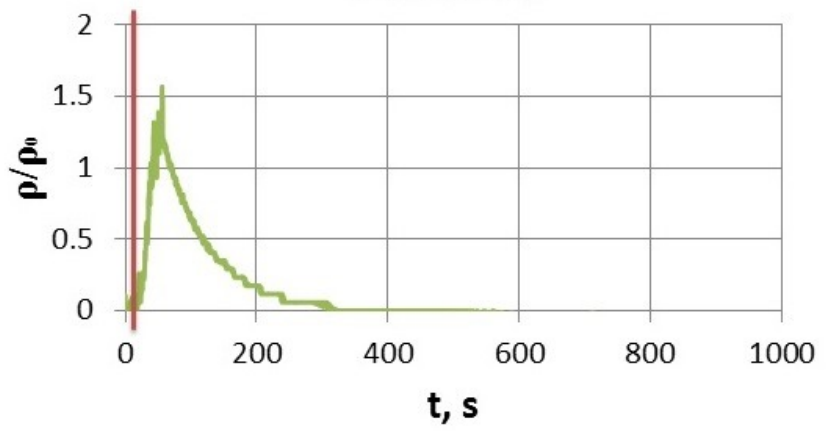

Fig.17. CSE factor exposure time dependence for K-RJ-4-3 sample in the case of $\mathrm{R}-\mathrm{R}$ recording polarizations.

\section{L-R K-RJ-4-3}

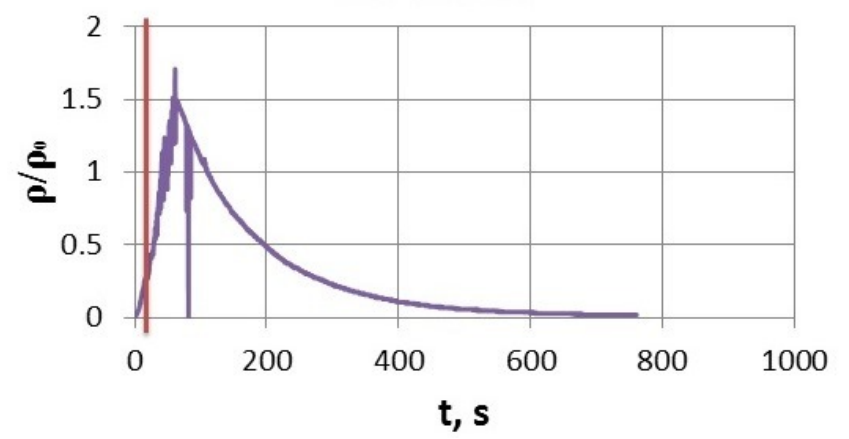

Fig.19. CSE factor exposure time dependence for K-RJ-4-3 sample in the case of L-R recording polarizations.

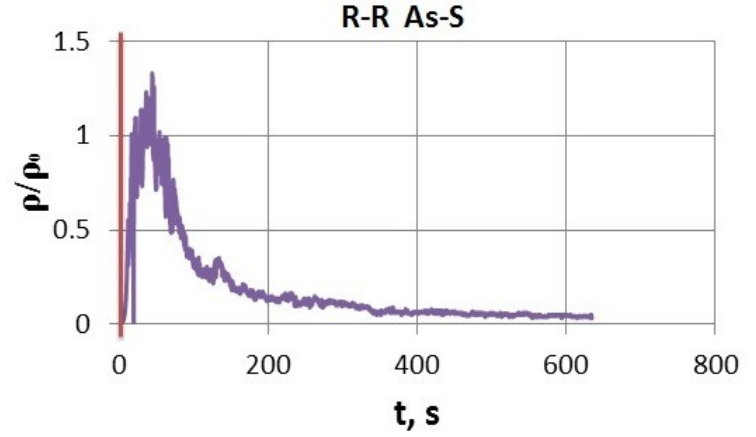

Fig.18. CSE factor exposure time dependence for As-S sample in the case of $\mathrm{R}-\mathrm{R}$ recording polarizations

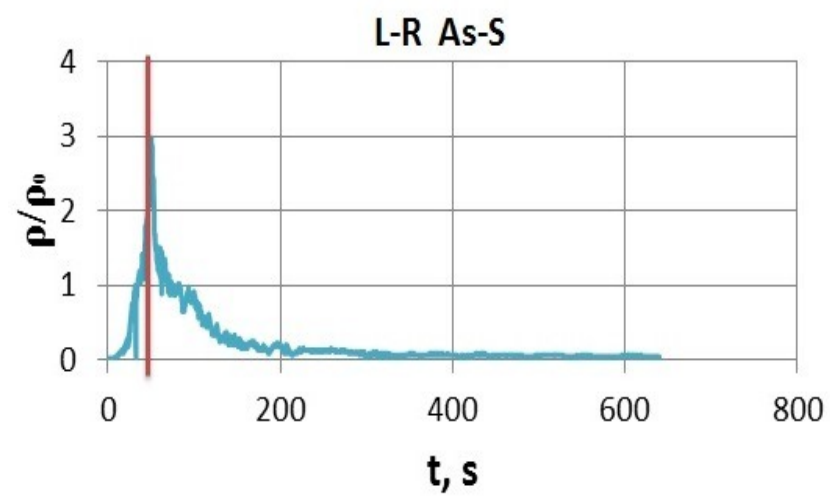

Fig.20. CSE factor exposure time dependence for As-S sample in the case of L-R recording polarizations

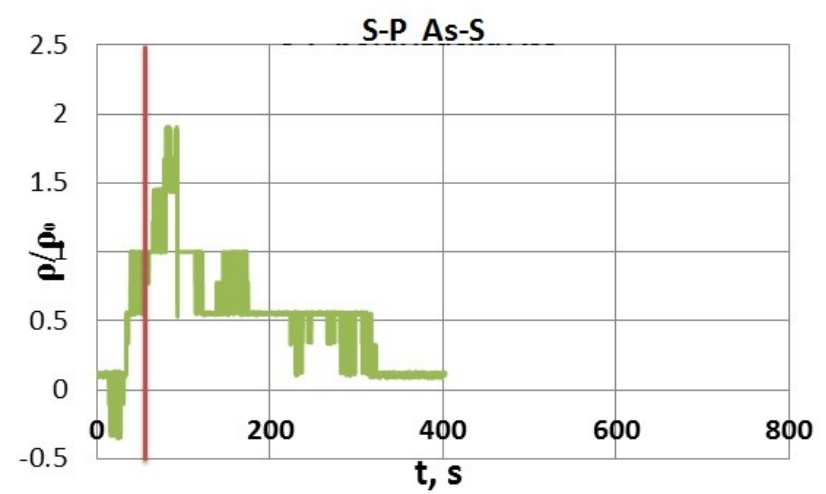

Fig.21. CSE factor exposure time dependence for As-S sample in the case of $s$ - $p$ recording polarizations. No CSE with these polarizations were observed in the case of K-RJ-4-3 sample.

CSE factor exposure time dependence is not presented for K-RJ-4-3 sample because no CSE was observed. DFWM efficiency for K-RJ-4-3 sample with $s-p$ polarizations was also much lower than in normal recording case (see Tables 1 and 3). Evidently, the effect of additional reflection HG is the least favourable for these recording polarizations 
Holographic parameters in the DFWM CSE geometry are summarized in Tables 5 and 6 . There, two exposure times are given $\left(t_{1}\right.$ and $\left.t_{2}\right)$ corresponding to the first recording stage (its end is marked with a vertical line in Figs. 13-21) with two incident beams $\mathrm{P}_{1}$ and $\mathrm{P}_{2}$ until the initial DFWM effieciency $\rho_{0} \approx 0.1 \rho_{\max }\left(\rho_{\max }\right.$ was taken from Tables 3,4), and to the second recording stage with blocked $\mathrm{P}_{1}$ beam (Fig.2), respectively.

Table 5. DFWM CSE holographic recording parameters of sample K-RJ-4-3 for different recording beam polarization pairs.

\begin{tabular}{|c|c|c|c|c|c|}
\hline & $s-s$ & $p-p$ & $\mathrm{R}-\mathrm{R}$ & $s-p$ & L-R \\
\hline$\left(\rho / \rho_{0}\right)_{\max }$ & 6.8 & 1.7 & 1.6 & - & 1.7 \\
\hline$P_{\max }, \%$ & 0.12 & 0.65 & 0.12 & - & 2.5 \\
\hline$t_{1} \mathrm{~s}$ & 25 & 60 & 25 & - & 40 \\
\hline$t_{2}, \mathrm{~s}$ & 70 & 100 & 50 & - & 50 \\
\hline$H_{\max }, \mathrm{J} /\left(\mathrm{cm}^{2} \%\right)$ & 440 & 125 & 330 & - & 17 \\
\hline $\begin{array}{l}\mathrm{REF} \\
\left(\% \mathrm{~cm}^{2}\right) / \mathrm{J}\end{array}$ & $2.7 * 10^{-4}$ & $5.2^{2} 10^{-3}$ & $3.7 * 10^{-4}$ & & 0.15 \\
\hline
\end{tabular}

Using $\mathrm{REF}_{\mathrm{CSE}}$ criterion one can see from Table 5 that the most efficient polarization pair is orthogonal L-R. Then come $p-p, \mathrm{R}-\mathrm{R}, s-s$ and $s-p$. This polarization dependence is almost the same as in DFWM case (Table 3).

Table 6. DFWM CSE holographic recording parameters of sample As-S for different recording beam polarization pairs.

\begin{tabular}{|c|c|c|c|c|c|}
\hline & $s-s$ & $p-p$ & $\mathrm{R}-\mathrm{R}$ & $s-p$ & $\mathrm{~L}-\mathrm{R}$ \\
\hline$\left(\rho / \rho_{0}\right)_{\max }$ & 1.2 & 3.0 & 1.3 & 0.8 & 3.0 \\
\hline$P_{\max }, \%$ & 0.73 & 0.15 & 20 & 0.009 & 0.57 \\
\hline$t_{l,} \mathrm{~s}$ & 5 & 40 & 50 & 50 & 30 \\
\hline$t_{2}, \mathrm{~s}$ & 10 & 80 & 70 & 7250 & 50 \\
\hline$H_{\max }, \mathrm{J} /\left(\mathrm{cm}^{2} \%\right)$ & 11 & 420 & $7.9^{*} 10^{-3}$ & $1.2^{*} 10^{-6}$ & $6.8^{*} 10^{-3}$ \\
\hline $\begin{array}{l}\mathrm{REF} \\
\left(\% \mathrm{~cm}^{2}\right) / \mathrm{J}\end{array}$ & $6.6^{*} 10^{-2}$ & $3.6^{*} 10^{-4}$ & & & \\
\hline
\end{tabular}

Using $\mathrm{REF}_{\mathrm{CSE}}$ criterion one can see from Table 6 that the most efficient polarization pair is scalar $s$ - $s$. Then come L-R, $\mathrm{R}-\mathrm{R}, p-p$ and $s-p$. This polarization dependence again is almost the same as in DFWM case (Table 4).

The following conclusions can be made from Tables 5 and 6 . The highest CSE factor of 6.8, the highest values of $\rho_{\max }$ and $\mathrm{REF}_{\mathrm{CSE}}$ are achieved in the case of sample K-RJ-4-3, contrary to the previous results. This material seems more suitable for DFWM CSE recording. The comparison of Tables 3 and 5, 4 and 6 reveals that generally DFWM CSE recording is less efficient than DFWM recording in both materials. Exception is $s-s$ case in sample K-RJ-4-3 when lower specific recording energy $H_{\max }$ and higher $\mathrm{REF}_{\mathrm{CSE}}$ are achieved. This means that under certain circumstances DFWM CSE recording can be more efficient than a simple DFWM recording. When results in Tables 5 and 6 are compared with the results recently obtained for CSE of normal two-wave HG recording in other molecular glasses ${ }^{16}$ one can say that the results are comparable. Much better CSE results in the case of normal HG recording are obtained in a- $\mathrm{As}_{2} \mathrm{~S}_{3}$ films (CSE factor up to $\left.1000, H_{\max }=2.5 \mathrm{~J}\left(/ \mathrm{cm}^{2} \%\right), \mathrm{REF}_{\mathrm{CSE}}=16(\% \mathrm{~cm})^{2} / \mathrm{J}\right)^{17}$. From the obtained experimental results one can conclude that azobenzene molecular glasses are promising materials for efficient DFWM CSE HG recording.

\section{CONCLUSIONS}

Polarization holographic grating (HG) recording in DFWM geometry and their coherent self-enhancement ( i.e., HG recording in DFWM CSE geometry ) are experimentally studied in azobenzene molecular K-RJ-4-3 film and in amorphous chalcogenide $\mathrm{As}_{2} \mathrm{~S}_{3}$ (As-S) film. The possibility of DFWM CSE recording is experimentally confirmed, for the first time to our knowledge. Normal HG recording in usual two-wave mixing geometry is experimentally studied as well for comparison.

When different HG recording modes are compared according to their recording efficiency factors (REF, see Section 2) then the DFWM recording is the most efficient in K-RJ-4-3 film (followed by DFWM CSE and normal HG recording) whereas normal two-wave HG recording is most efficient in As-S film (followed by DFWM and DFWM CSE recording). The highest DFWM efficiency of $14.5 \%$ and the highest DFWM CSE factor of 6.8 were achieved in K-RJ-43 film. However, As-S film was more efficient than K-RJ-4-3 film in all modes except for DFWM CSE recording. 
Azobenzene molecular glassy films are suitable materials for DFWM and DFWM CSE recording. In the particular case of $s-s$ recording polarizations DFWM CSE recording was even more efficient than DFWM recording.

Strong but different HG recording polarization dependences have been observed in both studied materials. They also depended on recording mode. In the case of K-RJ-4-3 film orthogonal L-R recording polarizations were most efficient in DFWM and DFWM CSE recording modes, but orthogonal $s-p$ polarizations were the most efficient in normal two-wave HG recording mode. In the case of As-S film scalar $s$-s polarizations were the most efficient in all HG recording modes.

It should be stressed that different geometry of DFWM and DFWM CSE recording modes leads to several distinctions in recording properties when compared to normal two-wave mixing recording mode. Not only recording efficiency and polarization dependence generally changes but also the shape of kinetic curves. Evidently, this is the result of additional reflection $\mathrm{HG}$ recorded by pump and probe beams.

Hologram recording in DFWM geometry (and in its extension - DFWM CSE geometry) has , at least, three advantages when compared to normal two-wave hologram recording: 1) possibility of real-time phase conjugation of the incident wave front is possible; 2) an object beam shutter is not necessary to measure the recording efficiency in real time; 3 ) a higher signal-to-noise ratio at the photodetector site.

\section{ACKNOWLEDGEMENTS}

The financial support of this work by the Latvian State Program Multifunctional materials project is greatly acknowledged.

\section{REFERENCES}

[1] Bratfalean R.T., Lloyd G.M., and Ewart P., „,Degenerate four-wave mixing for arbitrary pump and probe intensities", J.Opt.Soc.Am.B 16 (6), 952-960(1999).

[2] Yariv,A, „Four wave nonlinear optical mixing as real time holography”, Optics Communications 25 (1), 23-25 (1978).

[3] Gruzintsev, A.N. and Volkov, V.T., „Inversion of wave-front upon optically excited ZnO surface”, Physics and Technology of Semiconductors 38 (5), 543-546 (2004) (In Russian).

[4] Zeldovich, B.Ya, Pilipetsky, N.F and Shkunov V.V,[Wave Front Reversal], Nauka, Moscow, 19-29(1985)(In Russian).

[5] White, J.O. and Yariv, A., Optical Engineering 21(2), 224-229 (1982).

[6] Dmitryev, V.G. [Nonlinear Optics And Wave Front Reversal], Fizmatlit, Moscow, 7-16 (2003) (In Russian).

[7] Son, J.Y., Bobrinev, V.I., Jeon, H.W., Cho, Y.H. and Eom, Y.S.,"Direct image transmission through a multimode optical fiber", Appl. Optics 35 (2), 273-277(1996).

[8] Ito,K. and Sakai, J.I., "Comparative study of direct image transmission through various optical fibers using the phase-conjugate wave", Jap. J. of Appl. Phys. 46 (8A), 5172-5177 (2007).

[9] Lerosey, G., Rosny, J.de,, Tourin, A. and Fink, M., "Focusing beyond the diffraction limit with far-field time reversal", Science 315, 1120-1122 (2007).

[10] Yang, M. and Gu, C., "Application of phase-conjugate mirrors during recording of volume holographic memories", Appl. Optics 38(5), 855-862 (1999).

[11] Bouchal , Z. and Perina, J.,Jr., "Vectorial phase conjugation in four-wave mixing", Proc. SPIE 3820 (1999), doi:10.1117/12.353066.

[12]Lin, Z., Alcorn,T., Loncar, M., Johnson, S.G., and Rodriguez, A.W., "High-efficiency degenerate four-wave mixing in triply resonant nanobeam cavities", Phys. Rev A 89, 053839-1- 053839-10 (2014).

[13] Sing, B.P., Prasad, P.N., and Karasz, F.E., "Third-order non-linear optical properties of oriented films of poly( $p$-phenylene vinylene) investigated by femtosecond degenerate four wave mixing", Polymers 29(11), 1940-1942 (1988). 
[14] Ozols, A., Ozols, K., and Ivanovs, G., "Phase conjugation properties of a-As-S-Se films", Proc. SPIE 6180, 618010-1-618010-6 (2006).

[15].Saharov, D. and Ozols, A.," DFWM of focused laser beams in a- $\mathrm{As}_{2} \mathrm{~S}_{3}$ and azobenzene oligomer films", J.Mater.Sci:Mater.Electron, doi 10.1007/s10854-008-9639-8(2008).

[16] Ozols, A., Kokars, V., Augustovs, P., Malinovskis, D., Traskovskis, K., Zarins, E., and Ivanovs, G., "Selfenhancement of scalar and vector holographic gratings in azobenzene molecular glassy films", Optics and Photonics Journal 4, 143-152 (2014).

[17] Ozols, A., Nordman, N., Salminen, O., and Riihola, P., "Holographic recording in amorphous semiconductor films", Proc. SPIE 2968, 282-291 (1997).

[18] Shpotyuk, O.I., "Photostructural transformations in amorphous chalcogenide semiconductors", Phys. Stat.Sol.(b) 183, 365-374 (1994). 